\title{
Ländliche Gentrifizierung. Aufwertung und Verdrängung jenseits der Großstädte - Vorschlag für ein Forschungsprogramm
}

\author{
Michael Mießner and Matthias Naumann \\ Institut für Geographie und Regionalforschung, Universität Klagenfurt, 9020 Klagenfurt, Österreich \\ Correspondence: Michael Mießner (michael.miessner@aau.at)
}

Received: 28 October 2020 - Revised: 4 February 2021 - Accepted: 12 April 2021 - Published: 5 May 2021

\begin{abstract}
Kurzfassung. Not only since the Covid-19 pandemic, rural areas have received new attention as supposedly healthier and attractive places of residence. Regions previously characterized as shrinking are experiencing a highly selective influx of urban middle-class households and an increase in real estate and rental prices. These influxes and housing market developments raise the question of value increase and displacement. English-speaking, and especially British, human geographers have been studying the phenomenon of "rural gentrification" for several decades. This article therefore aims to systematize this state of the art in terms of its conceptual framework and empirical objects. Based on this, the article explains possible connections for German research on rural gentrification and discusses starting points for future research.
\end{abstract}

\section{Einleitung}

„Das Dorf muss gentrifiziert werden“ - mit dieser Forderung warb der Bevölkerungsforscher Reiner Klingholz in der „Frankfurter Allgemeinen Zeitung“ für eine gezielte Aufwertung ländlicher Räume (Lembke, 2019). Aktuell zeigen zahlreiche Medienberichte (z.B. Bätzing, 2020; Witzeck, 2020) ein wiedererwachendes Interesse an ländlichen Regionen als Wohnstandort in Zeiten der Covid-19-Pandemie. Der deutsche Rat der Immobilienweisen (2020) hat in seinem jüngsten Gutachten aufgezeigt, dass die Immobilienund Mietpreise in allen ländlichen Regionen gestiegen sind, in demographisch wachsenden ländlichen Kreisen lag die Steigerungsrate sogar über dem gesamtdeutschen Durchschnitt. Studien zur Entwicklung von Immobilienpreisen in ländlich geprägten Räumen im Umland von Großstädten (Braunschweig, 2019), Klein- und Mittelstädten in Thüringen (Schönig, 2020) oder zu zunehmenden Stadt-UmlandWanderungen (BiB, 2020) scheinen einen Trend hin zur Wiederentdeckung ländlicher Räume zu bestätigen, der sich auch in einem Bevölkerungswachstum einer Vielzahl ländlicher Räume ausdrückt (Thünen-Institut für Ländliche Räume, 2020). Ähnlich wie Klingholz sieht auch die Mecklenburger AnStiftung in Gentrifizierung eine „Chance“ (Schmidt,
2017:138) für die ländliche Regionalentwicklung. Während Arbeiten zur Politikberatung Gentrifizierung als eine Strategie der regionalen Entwicklung verstehen, hat sich in der Gentrifizierungsforschung eine andere Definition etabliert, die Gentrifizierung als immobilienwirtschaftliche Aufwertung und damit einhergehende Verdrängung einkommensniedriger Bevölkerungsgruppen konzeptionalisiert (Smith, 2019 [1979]; Slater, 2006). Hinsichtlich der Ursachen dieser Prozesse gibt es unterschiedliche Einschätzungen: Den einen gilt der Wandel von Konsumpräferenzen (Hamnett, 1991) und damit der Zuzug von sogenannten Gentrifiern als Auslöser, die anderen betonen die renditegetriebenen Investitionen als Treiber von Gentrifizierung (Smith, 2019 [1979]).

Während die mediale Behandlung ländlicher Gentrifizierung im deutschsprachigen Raum noch relativ neu ist, sich die Gentrifizierungsforschung auf urbane Räume beschränkt und die Forschungen zu ländlichen Räumen bisher nur einzelne, eher explorative Arbeiten (Krüger, 1995; Pichler, 1998; Schmied, 2002; Lier, 2012; Reichert-Schick, 2017) zum Thema enthalten, gibt es in der englischsprachigen $\mathrm{Hu}-$ mangeographie bereits seit mehreren Jahrzehnten eine rege Debatte um „rural gentrification“ (siehe für einen Überblick die Themenhefte in „Dialogues in Human Geography“, 2018 und in „Planning Theory \& Practice“, vgl. Scott et al., 2011). 
Den Begriff ländliche Gentrifizierung nutzen wir im Anschluss an Phillips et al. (2008:55), ,to refer to processes whereby middle or service class households are moving into villages and displacing local, working class groups, and often in the process also refurbishing, extending and converting properties". In der britischen Debatte ist dabei ein romantisiertes Verständnis von „,countryside“ als Sehnsuchtsort weißer Mittelklassen zentral (Smith und Phillips, 2001:458). Da das Konzept der Gentrifzierung anhand von städtischen Entwicklungen konzipiert wurde, stellt sich die Frage, ob eine Übertragung des Konzeptes auf ländliche Räume sinnvoll ist. Insbesondere, da ländliche Wohnungsmärkte deutlich stärker durch Wohneigentum geprägt sind, kann erwartet werden, dass eines der Kernmerkmale von Gentrifizierung - die Verdrängung - weniger verbreitet ist. So können Mieter*innen durch Mieterhöhungen, Modernisierungen, Klagen auf Eigenbedarf etc. leichter vertrieben werden als es bei Hauseigentümer*innen der Fall ist. Tatsächlich wurde die Frage von Verdrängung in der ,rural gentrification“-Debatte bisher weitgehend vernachlässigt, wie aktuell auch Phillips et al. (2021:72) einräumen. Dennoch konnten einige Studien nachweisen, dass einkommensschwächeren Bevölkerungsgruppen der Zugang zu Mietwohnungen und -häusern aufgrund von Preissteigerungen in ländlichen Räumen erschwert wurde (z.B. Solana-Solana, 2010). Dieser Ausschluss vom Zugang zu Wohnraum kann im Anschluss an Marcuse (1985:206) als ,exclusionary displacement" verstanden werden. Auch sein Konzept des Verdrängungsdruckes (1985:207), also die Aufwertung umliegender Gebäude und lokaler Dienstleistungen, die zukünftig zu Preissteigerungen führen können, kam in vorliegenden Studien zu ländlicher Gentrifizierung zur Anwendung (Smith und Higley, 2012). Aufwertung und Verdrängung sind damit Phänomene, die auch in ländlichen Kontexten festgestellt werden können.

Der Beitrag möchte die mittlerweile recht umfangreiche englischsprachige Debatte zu ,rural gentrification“ mit der aktuellen Aufmerksamkeit hinsichtlich einer möglichen Aufwertung ländlicher Räume in der Bundesrepublik verbinden. Wir adressieren dabei eine doppelte Leerstelle in der deutschsprachigen Debatte. Während zum einen die Gentrifizierungsforschung im deutschsprachigen Raum auf Städte fokussiert ist und ländliche Räume vernachlässigt hat, wird zum anderen Gentrifizierung in der Forschung zu ländlichen Räumen bis auf wenige Ausnahmen (Schmied, 2002; Reichert-Schick, 2017) nicht beachtet. Daraus folgen drei Ziele des Beitrags: Erstens geben wir einen komprimierten Überblick über die englischsprachige Forschung zu ländlicher Gentrifizierung und deren konzeptionellen Ansätze und empirischen Gegenstände. Zweitens zeigen wir mögliche Anschlüsse der deutschsprachigen Gentrifizierungsforschung und der Arbeiten zu ländlicher Entwicklung an die englischsprachigen Debatten zu ländlicher Gentrifizierung auf. Darauf aufbauend, leiten wir drittens erste Vorschläge für ein Forschungsprogramm zu Aufwertungs- und Verdrän- gungstendenzen in ländlichen Regionen im deutschsprachigen Raum ab. Der Beitrag schließt damit an Arbeiten an, die eine stärkere Verknüpfung der deutschsprachigen Forschung zu ländlicher Entwicklung mit internationalen Debatten verfolgen (Maschke et al., 2020). Unser Vorgehen beruht auf einer Auswertung der englischsprachigen Literatur, die Fragen von „rural gentrification“ behandelt. Die vorliegenden Arbeiten strukturieren wir hinsichtlich ihrer theoretischen Zugänge und ihres empirischen Vorgehens, um thematische Schwerpunkte, aber auch Unterschiede innerhalb der Debatte aufzuzeigen. Die in der internationalen Debatte wie auch in unserem Beitrag verwendeten Kategorien ,städtisch“ bzw. „ländlich“ verstehen wir dabei nicht als feststehende Abgrenzungen, sondern als immer wieder neu zu hinterfragende Bezeichnungen, die in enger Wechselwirkung zueinander stehen (Helbrecht, 2014). Der Beitrag schließt daran an und versteht ländliche Entwicklung nicht als losgelöst, sondern eng verknüpft mit städtischen Veränderungen wie auch mit anderen gesellschaftlichen Transformationen. Wenn die Aufwertung ländlicher Räume als Wohnstandort zu einem politischen Programm werden sollte (Schmidt, 2017; Lembke, 2019) und das Interesse am Wohnen in ländlichen Regionen anhält, könnten ländliche Regionen im deutschsprachigen Raum tatsächlich in naher Zukunft mit Gentrifizierungsprozessen und den damit verbundenen Konflikten und Verdrängungsprozessen konfrontiert sein. Auch wenn momentan die demographische Stabilisierung ländlicher Regionen im Vordergrund politischer Bemühungen steht, erscheint es lohnenswert, sich frühzeitig mit Prozessen der Aufwertung und Verdrängung auseinanderzusetzen und von den Erfahrungen aus anderen ländlichen Kontexten zu lernen.

\section{Konzeptionelle Ansätze zur Erklärung ländlicher Gentrifizierung}

Seit Ruth Glass' (1964) Arbeit zum Wandel Londoner Arbeiterviertel, mit der sie den Begriff der Gentrification prägte, ist Gentrifizierung zu einem wichtigen Untersuchungsgegenstand der Stadtforschung geworden. Lange Zeit galt Gentrifizierung vor allem als ein urbanes Phänomen, obwohl bereits seit den 1980er Jahren der Begriff der ländlichen Gentrifizierung verwendet (z.B. Parsons, 1980; Little, 1987) und ein Austausch von ortsansässiger Bevölkerung durch zuziehende Mittelschichtshaushalte in ländlichen Räumen nachgewiesen wurde (vgl. Robinson, 1996). Die Forschung zur ländlichen Gentrifizierung ist durch eine Vielzahl an Fallstudien geprägt (Nelson und Nelson, 2011), die unterschiedlichen Erklärungsansätzen für Gentrifizierung folgen.

Insbesondere nach Neil Smiths (2019 [1979]) früher Intervention in die Gentrifizierungsdebatte, in der er argumentierte, dass die bestehenden Erklärungsansätze innerstädtischer Gentrifizierung im Kern die Konsumpräferenzen von Menschen betonen und entsprechend nachfrageorientiert seien, gibt es eine ausgeprägte Debatte über die 
Gründe von Gentrifizierung. Die Erklärungen werden vor allem in konsumorientierte Ansätze, ,who stress the key role of choice, culture, consumption and consumer demand" und produktionsorientierte bzw. politisch-ökonomische unterschieden, ,who stress the role of capital, class, production and supply“ (Hamnett, 1991:174). Konsumorientierte Erklärungsansätze führen Gentrifizierung auf verändertes Konsumverhalten sowie auf veränderte Lebensstile, kulturelle Identitäten, Bildung, Gender und Sexualität zurück (Lees et al., 2008:89ff.). Die produktionsorientierten bzw. politischökonomischen Ansätze hingegen verstehen Gentrifizierung als Teil der kapitalistischen Produktion gebauter Umwelt und räumlich ungleicher Entwicklung. Die ökonomische und politische Produktion von ,rent gaps“ - verstanden als die Lücke zwischen aktuell realisierter und potenziell erzielbarer Grundrente - bildet demnach die Ursache für die immobilienwirtschaftliche Aufwertung von Quartieren und damit einhergehende Verdrängung (Smith, 1996). Die Differenzierung zwischen konsum- und produktionsorientierten Erklärungsansätzen lässt sich auch in der Forschung zu ländlicher Gentrifizierung nachvollziehen (Guimond und Simard, 2010), wobei ,the bulk of this literature emphasizes consumption as the motor of rural gentrification“ (Darling, 2005:1017). Diese Unterteilung der Erklärungsansätze ländlicher Gentrifizierung stellen wir im folgenden Abschnitt genauer dar.

\subsection{Konsumorientierte Erklärungen ländlicher Gentrifizierung}

Konsumorientierte Erklärungsansätze ländlicher Gentrifizierung konzentrieren sich auf veränderte Nachfrage nach Immobilien und sich wandelnde Bevölkerungsstrukturen. Vor dem Hintergrund einer postindustriellen Gesellschaft entsteht eine neue Mittelschicht, die Idealen von „Authentizität“ und individuellen Lebensstilen folgt (Hines, 2010b).

Ländliche Gentrifizierung geht demnach auf den Zuzug von Mittelschichtshaushalten in ländliche Gemeinden zurück (Newby, 1979), die durch attraktive Kulturlandschaften geprägt sind und entsprechend von Städter*innen als ländliches Idyll identifiziert werden (Halfacree, 1996). Diese Wanderungsbewegungen wurden bereits im Rahmen der Diskussion um Counterurbanisierung (z.B. Spencer, 1997) untersucht. Diese Arbeiten konstatierten einen Dekonzentrationsprozess von Bevölkerung (Berry, 1976:16), der sowohl mit Suburbanisierung als auch mit Bevölkerungszuwächsen in weiter entfernten ländlichen Räumen einherging. Entwicklungen von Counterurbanisierung wurden jedoch erst in den 1990er Jahren mit ländlicher Gentrifizierung in Verbindung gebracht (Phillips, 2010).

Für ländliche Gentrifizierung aus konsumorientierter Perspektive ist insbesondere der Zuzug von Gentrifiern von Bedeutung. Mit dem Zuzug von Gentrifiern in ländlichen Räumen sind Veränderungen der Konsummuster und -präferenzen verbunden, die auch die Siedlungsstruktur in ländlichen Räumen ganz erheblich verändern können
(Ghose, 2004). So werden neue Einfamilienhäuser gebaut (Reichert-Schick, 2017), Hausboote genutzt (Smith, 2007) oder neue Gebäude außerhalb der Ortskerne errichtet (Ghose, 2004). In der ländlichen Gentrifizierungsforschung werden verschiedene Gentrifier identifiziert, die unterschiedlich Einfluss auf ländliche Räume nehmen. Smith (2007) findet Hinweise, auf die schon frühzeitig in der Stadtforschung ausgemachten „marginal gentrifiers“ (Rose, 1984) - Personen, die nicht über ein großes Einkommen verfügen, häufig prekär beschäftigt, aber gut ausgebildet sind - in ländlichen Orten. In anderen Studien wurden als weitere Gentrifier lesbische Migrantinnen (Smith und Holt, 2005), Familien, die zur Sicherung einer guten Bildung ihrer Kinder aufs Land ziehen (Smith und Higley, 2012), oder ältere Menschen, die ihren Lebensabend auf dem Land verbringen wollen (Gilg und Kelly, 1997), ausgemacht. Letzterer Prozess wird in der Literatur auch als ,geriatrification“ (ebd.) bezeichnet.

Wie bereits angeführt, wird der Zuzug von Gentrifiern sehr stark durch die kulturlandschaftliche Attraktivität ländlicher Räume befördert. Das imaginierte ländliche Idyll (Halfacree, 1996) zieht Mittelschichtshaushalte an, die wiederum ihre Vorstellungen von idyllischen Verhältnissen an ihrem neuen Wohnort durchzusetzen versuchen (Smith und Phillips, 2001). In der Debatte hat sich dafür der Begriff der ,greentrification“ durchgesetzt, womit der Zusammenhang aus Umweltveränderungen und Gentrifizierung betont wird (Smith, 2011). Smith und Phillips (2001) identifizieren zwei Arten von „Greentifiern“: die peripher lebenden „Greentifier“, gut ausgebildete heterosexuelle Paare in der Familiengründungsphase, die ein Eigenheim besitzen und das Leben in einsamen Naturräumen bevorzugen, und die dörflichen „Greentifier“. Letztere sind Paare, die die Familiengründungsphase bereits hinter sich haben und/oder eine Familiengründung ablehnen, ebenfalls ein Eigenheim besitzen und das gemeinschaftliche Leben in Dörfern schätzen. Eine andere Folge des gestiegenen Interesses an ländlichen Idyllen sind der zunehmende Tourismus und sich darauf ausrichtende lokale Ökonomien (Leebrick, 2015). Aufgrund der Attraktivität ländlicher Orte für den Tourismus ziehen verstärkt Mittelschichtsangehörige in ländliche Räume und entwickeln dort auf diesen Tourismus zugeschnittene ökonomische und kulturelle Angebote (Hines, 2010a), wie etwa Erlebnisbauernhöfe. Darüber hinaus sind auch die vermehrte Nutzung von Gebäuden in ländlichen Räumen als Ferien- oder Wochenendhäuser ein Phänomen ländlicher Gentrifizierung, das mit der kulturlandschaftlichen Attraktivität und touristischen Nutzungen in Verbindung gebracht wird (Visser, 2004). Die konsumorientierte Perspektive auf ländliche Gentrifizierung thematisiert, wie das Konsumverhalten von Gentrifiern ländliche Räume verändert. 


\subsection{Politisch-ökonomische Erklärungen ländlicher Gentrifizierung}

Politisch-ökonomische bzw. produktionsorientierte Ansätze betten ländliche Gentrifizierungsprozesse stärker in strukturelle ökonomische Veränderungen und räumlich ungleiche Entwicklungen ein. Hier lassen sich vor allem zwei Ursachen von ländlicher Gentrifizierung identifizieren: veränderte Investitionen in die gebaute Umwelt und die postproduktivistische Transformation ländlicher Räume ${ }^{1}$.

Politisch-ökonomische Ansätze verstehen ländliche Gentrifizierung als Folge renditegeleiteter Investitionen in die gebaute Umwelt. So zeigte Martin Phillips in einem Schlüsselaufsatz (1993:129f.), dass Gentrifier in ländlichen Räumen die Häuser zwar als Eigenheim nutzen und insofern auf den ersten Blick keine Renditeinteressen im Zentrum der Kaufentscheidung standen, sondern der Erwerb von Wohnraum zur Selbstnutzung. Allerdings macht Phillips deutlich, dass die Gentrifier mit einem wesentlich höheren Preis bei einem erneuten Verkauf kalkulieren, was durchaus auf Renditekalkulationen hinweist. Er konnte ebenso zeigen, dass die Sanierung von Gebäuden substantielle Steigerungen der Immobilienwerte zur Folge hatte. Dies interpretiert Phillips als Hinweis darauf, dass auch in ländlichen Räumen eine rent gap (Smith, 2019 [1979]) von entscheidender Bedeutung ist, die von Gentrifiern als ,effective embodiments of capital“ (Phillips, 1993:129) antizipiert und geschlossen wird. Gemeint ist damit, dass die Gentrifier „Charaktermasken“ (Marx, 1962[1867]:99), also „Personifikationen der ökonomischen Verhältnisse“ (ebd.), und dementsprechend ,kluge Investor*innen“ (Beauregard, 1986 zit. nach Phillips, 1993:129) sind, die Investitionen immer auch als Wertanlage sehen und bei einem möglichen Verkauf eine Rendite erzielen wollen.

Phillips macht darüber hinaus anschließend an die Einteilung der Klassen von Wright (1993 [1978]:61ff.) deutlich, dass die Gentrifier überwiegend den ,capitalist classes“ (1993:130) angehören und bezeichnet deshalb ländliche Gentrifizierung als „Klassenkolonisierung“. Die beiden Feststellungen, dass die Gentrifier als Investor*innen agieren und dass sie den „capitalist classes“ angehören, deuten darauf hin, dass auch in ländlichen Räumen die Zirkulation des Kapitals von entscheidender Bedeutung für ländliche Gentrifizierungsprozesse ist. In diese Richtung weist auch die Studie von Darling (2005). Sie zeigt nicht nur die Entstehung einer ländlichen rent gap auf, sondern macht einen weiteren bedeutenden Punkt politisch-ökonomischer Erklärungen ländlicher Gentrifizierung deutlich: die Rolle der (lokal-)staatlichen Politik. Darling argumentiert in ihrer Untersuchung, dass das Bau- und Planungsrecht von entscheidender Bedeutung ist, weil es nur in sehr wenigen Orten zusätzliches Bauland aus-

\footnotetext{
${ }^{1}$ Auch konsumorientierte Arbeiten führen Post-Produktivismus an. Allerdings benennen sie diese Entwicklung für gewöhnlich nur als einen Hintergrund ländlicher Gentrifizierung, ohne die Zusammenhänge mit ländlichen Immobilienmärkten herauszuarbeiten.
}

weist, sodass Bauflächen äußerst knapp sind. Aus diesem Grund sind die Immobilienpreise so stark gestiegen, dass sie weit über dem für lokale Einkommen leistbaren Niveau liegen (ebd.:1022) und deshalb einkommensniedrigen Bevölkerungsschichten der Zugang zum Wohnen erschwert wird. Auch wenn Darling es nicht explizit benennt, ist hier die Parallele zu ,exclusionary displacement“ (Marcuse, 1985:206) offensichtlich. Damit spielen Politik und Planung bei der Aufwertung ländlicher Räume eine wichtige Rolle. Mark Shucksmith bezeichnet Planer*innen auch als ,Agent*innen der Gentrifizierung“, die Interessen von einkommensstarken Eigentümer*innen unterstützen (2011:607).

Der Zusammenhang zwischen Prozessen der Kapitalakkumulation und ländlicher Gentrifizierung ist auch bei der zweiten Ursache ländlicher Gentrifizierung von großer Bedeutung: der post-produktivistischen Transformation ländlicher Räume (Darling, 2005). War die Landwirtschaft im Globalen Norden in den 1970er Jahren noch durch expansive Produktion gekennzeichnet, so werden seit den 1980er Jahren Flächen und Höfe wie auch Arbeiter*innen aus der landwirtschaftlichen Nutzung entlassen (Phillips, 2009). Dies hatte eine kapitalistische Entwertung ländlicher Räume zur Folge (ebd.), die zu neuen räumlichen Ungleichheiten führte. Eine Vielzahl ländlicher Räume ist seitdem durch ,a move simply from production to consumption“ (Vergunst, 2016:286) geprägt. Dazu zählen die Veränderungen von bislang agrarisch genutzten Landschaften für touristische Nutzungen oder Freizeitgestaltung, wie sie sich beispielsweise in touristischen Familienbauernhöfen oder Golfplätzen ausdrücken. Es findet aber auch eine Diversifizierung von Hofstrukturen statt, z.B. in Nebenerwerbslandwirtschaft, ökologischer Landbau etc. Ehemals produktives Agrarland wurde aus politisch-ökonomischer Perspektive im Zuge des Übergangs zum Post-Produktivismus zunächst zu unterkapitalisiertem Land im Sinne der rent gap-Theorie (Darling, 2005), das nun im Zuge von ländlichen Gentrifizierungsprozessen erneut in Wert gesetzt wird (Phillips, 2004). Der Zuzug von Mittelschichtshaushalten oder die touristische Nutzung dieser Orte ist somit als Wiederinwertsetzung ländlicher Räume zu verstehen. Die dargestellten konzeptionellen Zugänge zu ländlicher Gentrifizierung sind eng mit den jeweiligen empirischen Gegenständen verknüpft. Der folgende Abschnitt gibt nun einen Überblick über die empirischen Schwerpunkte in der englischsprachigen Debatte zu ländlicher Gentrifizierung.

\section{Empirische Arbeiten zu ländlicher Gentrifizierung}

Die englischsprachigen Arbeiten zu ,rural gentrification“ behandeln ein breites Spektrum an empirischen Gegenständen, die im Folgenden zu Schwerpunkten gruppiert und hinsichtlich der Untersuchungsregionen dargestellt werden. Dieser Überblick hat keinen Anspruch auf Vollständigkeit, sondern 
die Funktion, die Debatte entlang ihrer bisherigen Schwerpunkte zu strukturieren.

\subsection{Empirische Gegenstände von „rural gentrification“}

Die empirischen Gegenstände der Arbeiten zu ,rural gentrification" fassen wir im Folgenden anhand von zwei Schwerpunkten zusammen: erstens, sozialen Wandel und Konflikte, die mit ländlicher Gentrifizierung verbunden sind, und zweitens, Wandel von Landnutzungen und Mensch-UmweltVerhältnissen als Resultat ländlicher Gentrifizierung.

Erstens bilden die Veränderungen der bisherigen Sozialstruktur durch den Zuzug großstädtischer Mittelklasseangehöriger den häufigsten empirischen Gegenstand der Arbeiten zu ländlicher Gentrifizierung. So heben Cloke und Thrift (1987) den Klassencharakter der Migration in ländliche Räume hervor und postulieren zugespitzt, dass „the service class has colonised more and more rural areas" (ebd.:327). Den sozial stark selektiven Zuzug in ländliche Räume stellen eine ganze Reihe von Autor*innen in einen Zusammenhang mit „Amenity“ bzw. „Lifestyle Migration“ (z.B. Ghose, 2004), d.h. Zuzug von Menschen, die in der Lage sind, ihren Wohnort entsprechend ihrer Freizeitvorlieben bestimmen zu können. Hier zeigt sich der enge Bezug von ländlicher Gentrifizierung zu Fragen von Tourismus und dessen Auswirkungen (Leebrick, 2015). Hinweise auf eine „Supergentrification" liefern Befunde zur Entstehung eines neuen „Landjunkertums“ in ländlichen Räumen, das sich an traditionellen Freizeitaktivitäten orientiert (Heley, 2010), oder auch zur Entstehung von ländlichen „gated communities“ (Mamonova und Sutherland, 2015). Dem Zuzug von neuen Bewohner*innen stehen Prozesse der Marginalisierung und Exklusion (Smith, 2011) gegenüber. So zeigt Little (1987) in einer Studie zu Wiltshire (Großbritannien), dass der Zuzug von Mittelschichtshaushalten zu Lasten der einkommensniedrigen Bevölkerungsgruppen geht und die „gap between rich and poor" (ebd.:197) weiter aufgeht. Die Veränderungen der Sozialstruktur sind damit durchaus umstritten (Cloke und Thrift, 1987). Dirksmeier (2009) zeigt beispielsweise, dass städtische Lebensstile in ländlichen Räumen auch mit Konflikten verbunden sind. Die Linien dieser Konflikte, z.B. ,Zugezogene“ vs. „Alteingesessene“, zu untersuchen und zu hinterfragen, bildet ein wichtiges Anliegen kritischer Forschung zu ländlicher Gentrifizierung (Solana-Solana, 2010).

Die Aufwertung ländlicher Räume verändert zweitens auch gesellschaftliche Naturverhältnisse. Die vermeintliche „Idylle“ ländlicher Naturen bildet einerseits einen wichtigen Grund für den Zuzug in ländliche Räume (siehe Abschnitt 2.1), dies zeigt die Lage von gentrifizierten Regionen in der Nähe von Küsten, Nationalparks oder in weiteren als attraktiv wahrgenommenen Naturräumen. Andererseits transformieren die neuen Bewohner*innen bestehende Naturverhältnisse, indem sie Landschaften konsumieren und weniger als Ressourcen der Produktion verstehen (Ghose, 2004). Diese „greentification“ (Smith und Phillips, 2001) führt zu neuen Wahrnehmungen, aber auch praktischen Aneignungen ländlicher Natur (Richard et al., 2014). Hierzu zählen neben der Renovierung und Neugestaltung von Gebäuden die Anlage von Gärten (ebd.:7), aber auch neue landwirtschaftliche Nutzungen, die jedoch eher als „Hobby“ betrieben werden (Sutherland, 2012). Dieser Landnutzungswandel lässt sich auf die Differenz zurückführen zwischen der Rendite, die mit bisheriger landwirtschaftlicher Landnutzung und der Rendite, die mit neuen Nutzungsformen, zum Beispiel Tourismus, generiert werden kann (Darling, 2005:1022). Verdrängung durch ländliche Gentrifizierung umfasst damit nicht nur bisherige Bewohner*innen, sondern auch bisherige Landnutzungen. So ist auch der Landnutzungswandel verbunden mit Konflikten, z.B. um die Privatisierung bislang offen zugänglicher Flächen, der Kommodifizierung natürlicher Ressourcen oder die Vorgaben des Naturschutzes (Ghose, 2004). Hier werden häufig neue Bewohner*innen aktiv, die naturräumliche Vorzüge gegen weitere Zuzüge verteidigen (Bryson und Wyckoff, 2010:72). Die enge Verknüpfung ländlicher Gentrifizierung mit bestimmten Naturräumen steht für die lokale Differenzierung bei der Aufwertung ländlicher Räume, die sich auf ausgewählte Orte konzentriert. Dies zeigt sich auch bei den Untersuchungsregionen bisheriger Arbeiten.

\subsection{Untersuchungsregionen ländlicher Gentrifizierungsforschung}

Bei den Untersuchungsregionen, auf die sich die englischsprachige Debatte zu ländlicher Gentrifizierung bezieht, lässt sich eine Konzentration auf bestimmte Regionen feststellen (siehe Phillips und Smith, 2018 für einen Überblick von Arbeiten zu Großbritannien, Frankreich und den USA). Die Ursprünge der Debatte in Großbritannien zeigen sich in einer Vielzahl an Beiträgen zu England (z.B. Phillips, 2014), Schottland (z.B. Stockdale, 2010) oder Wales (z.B. Stockdale, 2014). Der Nordwesten der USA ist eine weitere Region, auf die sich viele Untersuchungen beziehen (z.B. Ghose, 2004). Für verschiedene europäische Länder, wie zum Beispiel Frankreich (z.B. Richard et al., 2014), Russland (z.B. Mamonova und Sutherland, 2015) oder Katalonien (z.B. Solana-Solana, 2010), liegen ebenfalls Arbeiten vor. Vor diesem Hintergrund plädiert Lopez-Morales (2018) dafür, auch den Globalen Süden in der Debatte zu ländlicher Gentrifizierung stärker zu berücksichtigen. Hier bieten sich Bezüge zu Arbeiten der Migrationsforschung an, die beispielsweise touristisch attraktive Bergregionen in Lateinamerika untersuchen (Kordel und Pohle, 2018).

Beim räumlichen Zuschnitt der vorliegenden Arbeiten fällt zum einen auf, dass Kleinstädte nur selten thematisiert werden, und zum anderen, dass der räumliche Fokus von ,ländlich" unklar bleibt. Darling (2005) spricht zum Beispiel in Abgrenzung zur ländlichen Gentrifizierungsdebatte in Großbritannien von „wilderness gentrification“ um darauf hinzuweisen, dass im Fall von Adirondack Park (USA) weniger 
die Charakterisierung als ländlicher Raum als vielmehr das Vorhandensein eines Nationalparks und die damit verbundenen staatlichen Regulierungen ausschlaggebend für die Gentrifizierung waren. So ist genauer zu bestimmen, ob ganze Landkreise, Gemeinden, Dörfer, Ortsteile oder Streusiedlungen gentrifiziert werden. Auch wäre kritisch zu hinterfragen, ob die Prozesse ländlicher Gentrifizierung in Anlehnung an Smith (2002) als Teil der ,global urban strategy“ oder, wie es Holm (2013) für Berlin formuliert hat, als „Gentrification Mainstream" bezeichnet werden können.

Gerade weil ländliche Gentrifizierung nicht überall und lokal sehr unterschiedlich stattfindet, bietet sie einen Zugang, die kleinräumige Differenzierung ländlicher Räume zu verstehen. Andererseits kann ländliche Gentrifizierung durchaus auch in Großstädten stattfinden, etwa in historischen Dorfkernen, die sich in Großstädten befinden. Heins spricht von der Entstehung einer ,pseudo-countryside, a residential environment with the characteristics of the countryside but not located there" (2004:39) aufgrund der Nachfrage nach ländlichem Wohnen. Beispiele hierfür wären „Stadtvillen“ oder „Townhouses“ in innerstädtischen Quartieren. Hier könnte auch ein Bezug auf die Entstehung von Mittelschichtsenklaven in Städten, von Susanne Frank als ,innere Suburbanisierung“ (2014) beschrieben, lohnenswert sein. Ein Blick auf historische „Künstlerkolonien“ im Umland von Berlin wie die Kleinstädte Bad Saarow (Landkreis Oder-Spree) und Buckow (Landkreis Märkisch Oderland) könnte Kontinuitäten oder Brüche in der Sehnsucht nach ländlichen Idyllen herausarbeiten. So kann ein Fokus auf Vorstellungen ländlicher Idyllen auch die städtische Gentrifizierungsforschung voranbringen (Bernt, 2018). Die genannten Beispiele im Berliner Umland machen deutlich, dass auch im deutschsprachigen Raum ländliche Gentrifizierungsprozesse einsetzen könnten. Die Anschlussmöglichkeiten für die deutschsprachige Forschung thematisiert der folgende Abschnitt.

\section{Anschlüsse für die deutschsprachige Forschung}

Die Arbeiten zu ländlicher Gentrifizierung bieten vielfältige Anknüpfungspunkte sowohl für die deutschsprachige Gentrifizierungsforschung, die bislang vor allem städtische Räume thematisierte als auch für die deutschsprachige Forschung zu ländlichen Räumen, die Fragen von Aufwertung und Verdrängung bislang nicht im Fokus hatte. Wie ländliche Gentrifizierung damit Anknüpfungspunkte für beide Forschungsstränge bilden kann, diskutiert der folgende Abschnitt.

\subsection{Anknüpfungspunkte für die deutschsprachige Gentrifizierungsforschung}

Für die deutschsprachige Gentrifizierungsforschung mit ihrer starken Verankerung in Städten bietet die Debatte zur ländlichen Gentrifizierung die Möglichkeit, den empirischen wie auch konzeptionellen Fokus auf Entwicklungen jenseits von urbanen Räumen zu erweitern.
Im Anschluss an konsumorientierte Erklärungsansätze in der deutschsprachigen Gentrifizierungsforschung (z.B. Blasius, 1993; Friedrichs und Kecskes, 1996) gibt es erste Arbeiten zu ländlicher Gentrifizierung. Doris Schmied (2002) untersuchte ländliche Gentrifizierung im Cotswold District in Südwestengland. Dabei konnte sie nachweisen, dass die Hoffnung auf ein ländliches Idyll ein zentrales Zuzugsmotiv wohlhabender Mittelschichtshaushalte darstellte, die in der Untersuchungsregion günstige Häuser kauften. Auch sie findet Hinweise auf ,marginal gentrifier“, die trotz vergleichsweise geringer Einkommen ländliche Gentrifizierung vorantreiben, und stellt Ruhestandswanderungen und eine Alterung fest, die jedoch auf dem Wegzug jüngerer Bewohner*innen aus der Region beruht. Zudem könnte der Zuzug von „Lifestyle Migrant*innen“ in ländlich periphere Regionen (Gruber et al., 2017) ländliche Gentrifizierungsprozesse auslösen. Die in der englischsprachigen Debatte etablierte Untersuchung möglicher Formen von ,greentification“ (Smith, 2011) könnte helfen, den Zusammenhang zwischen der ökologischen oder touristischen Überformung ländlicher Kulturlandschaften und dem sozialstrukturellen Wandel in ländlichen Regionen zu verstehen.

Die seit der Jahrtausendwende immer stärker rezipierte deutschsprachige Gentrifizierungsforschung, die auf politisch-ökonomische Erklärungsansätze aufbaut (z.B. Schipper, 2013; Holm, 2018), könnte ebenfalls um Fragen des Wandels ländlicher Immobilienmärkte erweitert werden. So sind insbesondere ländliche Küstenorte (Rohr, 2008) oder auch ländliche Räume in den Bergen (Job und Mayer, 2013) touristisch in Wert gesetzt worden und anschauliche Beispiele ländlicher Gentrifizierung post-produktivistischer Regionen. Politisch-ökonomische Ansätze betonen darüber hinaus die Bedeutung von Investor*innen für ländliche Gentrifizierung. Hier könnte zum einen die bisherige deutschsprachige Forschung zur Finanzialisierung von Wohnraum (Heeg, 2013) und zur Bedeutung von lokalen (Klein-)Investor*innen (Miessner, 2021) um Fragen ländlicher Wohnungsmärkte erweitert werden. Schipper (2013) legt am Beispiel von Frankfurt am Main eine überzeugende empirische Operationalisierung der rent gap-Theorie für den deutschen Kontext vor. Es gibt bisher - nicht nur im deutschsprachigen Raum - nur wenige systematische an der rent gap-Theorie orientierte räumliche Untersuchungen für Metropolregionen (z.B. Porter, 2010). Arbeiten, die über den engeren metropolitanen Verflechtungsraum hinausgehen, fehlen unseres Wissens. Die Arbeit von Darling (2005) zeigt aber, dass die rent gapTheorie auch auf ländliche Räume übertragbar ist, sodass eine systematische räumliche Untersuchung möglich scheint. Eine solche Forschung würde es ermöglichen, zu verstehen, wo und warum sich rent gaps öffnen. Damit könnte die räumliche Zirkulation des Kapitals durch die gebaute Umwelt nicht nur in Städten, sondern auch in ländlichen Kontexten nachvollzogen werden. Die politisch-ökonomischen Ansätze betonen darüber hinaus die Rolle von Regionalund Planungspolitiken für ländliche Gentrifizierungsprozes- 
se (Darling, 2005). Hieran anschließend könnte im deutschsprachigen Kontext untersucht werden, welche sozialräumlichen Wirkungen die Neuausrichtung staatlicher Raumplanungspolitik (Mießner, 2017) und der damit einhergehende verschärfte Wettbewerb zwischen Regionen (Kallert und Dudek, 2019) zeitigt. Der so durchgesetzte Fokus auf die „Innovationsleistung“ (Caprarese, 2007) im Zuge des „New Regionalism" folgt neoliberalen Logiken (Kröcher, 2008) und könnte den Zuzug einkommensstarker Bevölkerungsschichten befördern.

\subsection{Anschlüsse für die deutschsprachige Forschung zu ländlichen Räumen}

Die vorliegenden Arbeiten der englischsprachigen Humangeographie zu „rural gentrification“ bieten eine konzeptionelle Erweiterung der Forschung zu ländlichen Räumen um Prozesse der Gentrifizierung und können empirisch an Untersuchungen zu ländlicher Entwicklung im deutschsprachigen Raum anknüpfen.

In empirischer Hinsicht schließt die Debatte um ländliche Gentrifizierung an aktuelle Warnungen in der deutschen Presse an, die wegen steigender Bodenpreise von einer drohenden Vertreibung der ,eigentlichen Landbevölkerung“ (Bätzing, 2020) sprechen. Zudem zeigt eine erste Untersuchung, dass die Wohnungsfrage in kleineren Städten weniger eine quantitative Frage als eine Frage der Passfähigkeit zu bestimmten Bedürfnissen ist (Schönig, 2020). So ist beispielsweise für ältere Menschen nicht immer barrierearmer Wohnraum verfügbar. Hier bietet die englischsprachige Debatte um sozialen Wandel, Verdrängung und damit verbundene Konflikte erste Indizien, welche Konflikte auch in ländlichen Räumen Deutschlands zukünftig auftreten und wie diese empirisch untersucht werden könnten. Eine erste Untersuchung von Reichert-Schick (2017) zeigt die mit dem Zuzug aus Luxemburg verbundenen Veränderungen in der Obermosel entlang der Kategorien sozial-kultureller, baulicher, ökonomisch-infrastruktureller und symbolischer Wandel. Über diese bereits von deutschen Forscher*innen aufgegriffenen Fragen der Zuwanderung hinaus könnte ein Fokus auf den Klassencharakter der Migrationsbewegungen (Cloke und Thrift, 1987) wichtige Erkenntnisse über die sozialstrukturellen Veränderungen in ländlichen Räumen hervorbringen und zu einem tieferen Verständnis von Konflikten zwischen bisheriger Bevölkerung und Zuziehenden beitragen. Die Aneignung ländlicher Räume durch Angehörige der großstädtischen Mittelschicht kann auch als eine Form des „Land Grab“ verstanden werden (Steel et al., 2017), der eng mit städtischen und anderen gesellschaftlichen Entwicklungen verknüpft ist. Dies empirisch herauszuarbeiten, könnte eine weitere Aufgabe deutschsprachiger Forschungen sein.

Weiterhin zeigen die eingangs erwähnten Bestrebungen für eine Aufwertung ländlicher Regionen die politische Dimension ländlicher Gentrifizierung, die es auch in der Bundesrepublik zu untersuchen gilt. Hier wäre es lohnenswert, unterschiedliche Positionen und mögliche Konflikte zwischen, aber auch innerhalb der Landes- und Kommunalpolitik in den Blick zu nehmen. Das Wohnen auf dem Land ist schließlich auch ein Teil der „Infrastrukturen für "gleichwertige Lebensverhältnisse" (Simmank, 2020) in Deutschland. Darüber hinaus lassen sich Fragen des Landnutzungswandels, die mit "rural gentrification“ verfolgten Interessen unterschiedlicher Akteure und damit verbundene Konflikte mit aktuellen Diskussionen um die Gestaltung von ländlichen Energielandschaften (Bosch und Schmidt, 2020) verbinden.

Hinsichtlich der Untersuchungsregionen bietet der deutschsprachige Raum einerseits zahlreiche Beispiele für Regionen, die aufgrund naturräumlicher Reize als Beispiele für ländliche Gentrifizierung in Frage kommen. Andererseits bietet vor allem Ostdeutschland eine Gelegenheit, ländliche Räume, die eine umfangreiche Transformation erfahren haben, auf Hinweise einer Aufwertung zu untersuchen und damit neue Aspekte in die englischsprachige Debatte einzubringen. Die Übertragung des im Rahmen der „Kölner Gentrifizierungsstudien“ (Friedrichs und Blasius, 2016) entwickelten Wohnungspanels (Friedrichs und Blasius, 2015), das die Wohnung bzw. das Einfamilienhaus als Grundlage der Untersuchung sehr kleinräumiger Veränderungen nutzt, in Wellen über mehrere Jahre durchgeführt wird und so die Erforschung von Gentrifizierungsverläufen ermöglicht (Friedrichs und Blasius, 2016), könnte zum besseren Verständnis der unterschiedlichen Phasen ländlicher Gentrifizierungsprozesse beitragen. Eine Herausforderung wäre dabei jedoch die geringe Fallzahl, die in ein solches Panel in ländlichen Regionen einfließen würde. Aufbauend auf diese konzeptionellen und empirischen Anschlüsse für eine deutschsprachige Forschung an die Debatte zu ,rural gentrification" sollen im folgenden Kapitel mögliche Aspekte für ein Forschungsprogramm zu ländlicher Gentrifizierung benannt werden.

\section{Ländliche Gentrifizierung im deutschsprachigen Raum - ein Forschungsprogramm}

Die vorhergehenden Ausführungen machen deutlich, dass die Forschung zu ländlicher Gentrifizierung im deutschsprachigen Raum in konzeptioneller und empirischer Hinsicht an die englischsprachige Debatte anschließt, aber auch eigene Beiträge für deren Weiterentwicklung leisten könnte. Wir schlagen hierfür die folgenden Ausgangspunkte vor, an denen sich künftige Arbeiten zu ländlicher Gentrifizierung orientieren könnten.

Auf theoretisch-konzeptioneller Ebene wäre erstens zu fragen, ob die beschriebenen Erklärungsansätze für „rural gentrification" den Entwicklungen ländlicher Räume in Deutschland gerecht werden. Die englischsprachige Debatte um vergleichende Ansätze in der Stadtforschung (Robinson und Roy, 2016) hat zu einer Ausweitung der Untersuchungs- 
kontexte in der Gentrifizierungsforschung geführt (Bernt, 2016). Die Debatte macht aber auch deutlich, dass die Übertragung etablierter Theorieangebote auf andere Kontexte den jeweiligen Entwicklungen nicht immer gerecht wird. Daran anschließend wäre zu klären, ob die englischsprachigen Erklärungsansätze beispielsweise die spezifischen Transformationen in Ostdeutschland angemessen erfassen können. $\mathrm{Zu}-$ dem wäre kritisch zu reflektieren, ob die Übertragung des im städtischen Kontext entwickelten Gentrifizierungsbegriffes auf ländliche Kontexte sinnvoll ist. Schon seit einiger Zeit wird kritisiert, dass der Begriff der Gentrifizierung zu weit gedehnt (Lees et al., 2008) und seines ursprünglich kritischen Gehalts, dem Fokus auf die Verdrängung einkommensschwacher Bevölkerung, beraubt wurde (Slater, 2006). Entsprechend wäre zu fragen, wer Gewinner*innen und wer Verlierer*innen des ökonomischen, sozialen und ökologischen Wandels ländlicher Räume sind.

Eng verknüpft mit dieser Frage der Übertragbarkeit des Gentrifizierungsbegriffes auf ländliche Kontexte ist zweitens die Bedeutung von Verdrängung im ländlichen Kontext. Diese rückt jüngst stärker in den Fokus der Forschung (z.B. Phillips et al., 2021). Gerade weil die Wohnungsmärkte in ruralen Orten stark auf Eigentum und Selbstnutzung basieren, stellt sich die Frage, ob und wie hier Verdrängung beispielsweise durch Modernisierung stattfinden kann. Während Verdrängung in städtischen Räumen ein vergleichsweise kurzfristiger Prozess ist, konzipieren Phillips et al. (ebd.:74) Verdrängung als einen wesentlich längerfristigen Prozess von Desinvestition und späterer Reinvestition, bei dem Teile der Bevölkerung das Land aufgrund von Arbeitsplatzverlust etc. verlassen und neue zahlungskräftigere Klientel - durchaus Jahrzehnte später - zuzieht. So stellt die Konzeptionialisierung und empirische Untersuchung von Verdrängung eine wesentliche Herausforderung ländlicher Gentrifizierungsforschung dar.

Drittens ist die Abgrenzung ländlicher Gentrifizierung von anderen Formen der Gentrifizierung immer wieder neu zu bestimmen. In der Forschung gilt es daher beispielsweise zu zeigen, welche Prozesse sich der Suburbanisierung, welche ländlicher Gentrifizierung zuordnen lassen. Dies betrifft auch die Unterschiede von ländlicher Gentrifizierung und ,greentrification“ bzw. „environmental gentrification“ oder „geriatrification“. Hier könnte eine Typisierung unterschiedlicher Formen von Gentrifizierung anschließen, die sowohl in Städten wie auch ländlichen Räumen festzustellen sind.

Viertens stellen die Erfahrungen mit ländlichen Gentrifizierungsprozessen die gängigen Vorstellungen von Gentrifier zumindest teilweise in Frage. Zu klären wäre insofern, welche Personengruppen in ländlichen Räumen Deutschlands als Gentrifier auftreten, wie sich diese von städtischen Gentrifiern unterscheiden, welche Rolle sie in der Lokalpolitik spielen und was dies für die Konzeption des Phasenmodells der Gentrifizierung (Dangschat, 1988) bedeutet. So könnte aus der englischsprachigen Debatte die Frage aufgegriffen werden, ob Personen, die private Immobilien für die Selbst- nutzung erwerben, als Gentrifier verstanden werden können. Weiterhin könnten Erkenntnisse aus der Migrationsforschung in die Untersuchung der Motivationen für Zuwanderung in ländliche Räume einfließen.

Fünftens könnte die ländliche Gentrifizierungsforschung auch der deutschsprachigen kritisch-geographischen Forschung, die überwiegend auf städtische Kontexte fokussiert ist, neue Themen eröffnen. Die Diskussion um die Möglichkeiten und Grenzen kritischer Stadtforschung zur Untersuchung ländlicher Kontexte stellt nicht die zunehmende Durchlässigkeit der Kategorien von „Stadt“ und „Land“ infrage. Eine Untersuchung ländlicher Gentrifizierung kann dazu beitragen, zu prüfen, wie ,städtisch“ ländliche Räume mittlerweile sind, wie groß die Reichweite kritischer Stadtforschung ist und welche spezifischen Formen politischer Interventionen notwendig sind.

Auf empirischer Ebene wäre zunächst zu fragen, wo Gentrifizierung in ländlichen Räumen beobachtet werden kann. Damit ist der Bedarf nach Methoden verbunden, die diese Räume identifizieren können. Eine Inspiration könnte hierfür die „GentriMap“ (Holm und Schulz, 2018) für städtische Quartiere liefern. Hierfür wäre auch der Zusammenhang zwischen der Aufwertung ländlicher Immobilien und der Verdrängung einkommensschwacher Haushalte auf der einen Seite und der Restrukturierung ländlicher Ökonomien auf der anderen Seite genauer zu untersuchen. Der Strukturwandel in der Landwirtschaft, deren Beschäftigungswirkungen immer geringer werden, kann ein Ausgangspunkt für neu entstehende rent gaps und damit für die Wiederentdeckung ländlicher Immobilienmärkte sein. Der Bedeutungsgewinn von Tourismus als Erwerbsmöglichkeit in ländlichen Räumen ist mit einer Ausrichtung von Angeboten an den Interessen großstädtischer Nachfrager*innen verbunden.

Zweitens stellt sich das Problem der Verfügbarkeit von Daten zu ländlichen Immobilienmärkten und Sozialstrukturen. Sind schon Mietpreisdaten für kleinere Großstädte kaum verfügbar und müssen nicht selten erst eigens erhoben werden (Mießner, 2021), so stellt sich dieses Problem aufgrund geringerer Fallzahlen in Kleinstädten und ländlichen Räumen umso mehr. Aufgrund der häufig geringen Fallzahlen stellt sich eine solche Herausforderung auch für die Immobilienpreise kaufpreisarmer Lagen (Dorndorf et al., 2017). Ebenso sind sozialstatistische Daten bereits in Mittelstädten aus Datenschutzgründen nur selten kleinräumig differenziert verfügbar - für ländliche Regionen dürften diese Herausforderungen noch größer sein.

Drittens ist die städtische Situiertheit akademischer Wissensproduktion bei der Untersuchung ländlicher Gentrifizierung kritisch zu hinterfragen. Wissenschaftler*innen, die zu ländlicher Entwicklung forschen, sind häufig in Großstädten sozialisiert und arbeiten an Einrichtungen in Großstädten. Somit besteht die Gefahr, städtische Perspektiven auf ländliche Kontexte zu übertragen und etwaige Besonderheiten ländlicher Räume zu übersehen. Diese Herausforderung 
sollte bei der methodischen Konzeption der Untersuchung berücksichtigt werden.

Viertens müssen Wissenschaftler*innen, die zu ländlicher Gentrifizierung in Deutschland arbeiten, mit einer Ambivalenz umgehen. So ist die Forschung einerseits mit den Widersprüchen und Konflikten von ländlicher Gentrifizierung konfrontiert, die etwa bei der Aufwertung ländlicher Räume entstehen. Andererseits müssen sich Landforscher*innen auch der Frage stellen, was ihre konkreten Alternativen zu Abwanderung, Alterung und Peripherisierung jenseits des Zuzugs großstädtischer Mittelschichtshaushalte sind. Damit stellt sich die Aufgabe für eine Geographie ländlicher Räume, nach Perspektiven ländlicher Entwicklung zu suchen, die über die Optionen Auf- oder Abwertung hinausgehen. Hierfür kann eine deutschsprachige Forschung zu ländlicher Gentrifizierung erste Erkenntnisse hervorbringen.

Datenverfüg barkeit. Für diesen Artikel wurden keine Datensätze genutzt.

Autorenmitwirkung. Der Aufsatz wurde von den Autoren gemeinsam entwickelt und verfasst.

Interessenkonflikt. Die Autor*innen erklären, dass kein Interessenkonflikt besteht.

Danksagung. Wir danken Jan Glatter für die Kommentierung einer früheren Version dieses Aufsatzes sowie den beiden anonymen Gutachter*innen für die hilfreichen Hinweise zu unserem Beitrag.

Begutachtung. This paper was edited by Nadine Marquardt and reviewed by two anonymous referees.

\section{Literatur}

Bätzing, W.: „In Krisen ist die Flucht aufs Land ein typisches Phänomen“, in: Frankfurter Allgemeine Sonntagszeitung vom 5. April 2020.

Bernt, M.: Very particular, or rather universal? Gentrification through the lenses of Ghertner and López-Morales, City, 20, 637-644, https://doi.org/10.1080/13604813.2016.1143682, 2016.

Bernt, M.: Gentrification between Urban and $\mathrm{Ru}-$ ral, Dialogues Hum. Geogr., 8, 31-35, https://doi.org/10.1177/2043820617752001, 2018.

Berry, B.: Urbanisation and Counterurbanisation, SAGE, Beverly Hills, 1976.

BiB - Bundesinstitut für Bevölkerungsforschung: Raus aus der Großstadt: Stadt-Umland-Wanderung nimmt zu, Pressemitteilung vom 28. Mai 2020, Bundesinstitut für Bevölkerungsforschung, Wiesbaden, 2020.
Blasius, J.: Gentrification und Lebensstile: Eine empirische Untersuchung, Deutscher Universitätsverlag, Wiesbaden, 1993.

Bosch, S. and Schmidt, M.: Ungerechte Energielandschaften die Produktion von Raum im Kontext der Transformation des deutschen Energiesystems, Geogr. Helv., 75, 235-251, https://doi.org/10.5194/gh-75-235-2020, 2020.

Braunschweig, B.: Stadt-Land-Dualismus als Faktor erhöhter Flächeninanspruchnahme und angespannter Wohnungsmärkte?, in: Flächennutzungsmonitoring XI. Flächenmanagement - Bodenversiegelung - Stadtgrün (IÖR Schriften Nr. 77), Herausgeber: Meinel, G., Schumacher, U., Behnisch, M., und Krüger, T., Rhombos, Berlin, 159-196, 2019.

Bryson, J. und Wyckoff, W.: Rural gentrification and nature in the Old and New Wests, J. Cult. Geogr., 27, 53-75, https://doi.org/10.1080/08873631003593232, 2010.

Caprarese, M.: Räumliche Verteilung der Innovationsleistung in der Schweiz, Geogr. Helv., 62, 210-220, https://doi.org/10.5194/gh62-210-2007, 2007.

Cloke, P. und Thrift, N.: Intra-class conflict in rural areas, J. Rural Stud., 3, 321-333, https://doi.org/10.1016/0743-0167(87)900519, 1987.

Dangschat, J.: Gentrification: Der Wandel innenstadtnaher Wohnviertel, in: Soziologische Stadtforschung, Sonderheft 28/1988, Herausgeber: Friedrichs, J., Westdeutscher Verlag, Opladen, 272-292, 1988.

Darling, E.: The City in the Country: Wilderness Gentrification and the Rent Gap, Environ. Plan A, 37, 1015-1032, https://doi.org/10.1068/a37158, 2005.

Dialogues in Human Geography: Article Forum on Martin Phillips and Darren Smith "Comparative approaches to gentrification: Lessons from the rural”, Dialogues Hum. Geogr., 8, 3-58, 2018.

Dirksmeier, P.: Urbanität als Habitus. Zur Sozialgeographie städtischen Lebens auf dem Land, transcript, Bielefeld, 2009.

Dorndorf, A., Soot, M., Weitkamp, A., und Alkhatib, H.: Aggregation und Gewichtung von unterschiedlichen Wertermittlungsdaten in kaufpreisarmen Lagen mittels Varianzkomponentenschätzung, Allgemeine Vermessungsnachrichten, 5, 123-136, 2017.

Frank, S.: Innere Suburbanisierung als Coping-Strategie: Die ,neuen Mittelschichten“ in der Stadt, in: Urbane Ungleichheiten. Neue Entwicklungen zwischen Zentrum und Peripherie, Herausgeber: Berger, P., Keller, C., Klärner, A., und Neef, R., Springer VS, Wiesbaden, 157-172, 2014.

Friedrichs, J. und Blasius, J.: The Dwelling Panel - A New Research Method for Studying Urban Change, Raumforschung und Raumordnung, 73, 377-388, https://doi.org/10.1007/s13147015-0369-0, 2015.

Friedrichs, J. und Blasius, J.: Die Kölner Gentrification-Studien, in: Gentrifizierung in Köln: Soziale, ökonomische, funktionale und symbolische Aufwertungen, Herausgeber: Friedrichs, J. und Blasius, J., Barbara Budrich, Leverkusen-Opladen, 7-27, 2016.

Friedrichs, J. und Kecskes, R. (Hrsg.): Gentrification: Theorie und Forschungsergebnisse, VS Verlag für Sozialwissenschaften, Wiesbaden, 1996.

Ghose, R.: Big Sky or Big Sprawl? Rural Gentrification and the Changing Cultural Landscape of Missoula, Montana, Urban Geogr., 25, 528-549, https://doi.org/10.2747/02723638.25.6.528, 2004. 
Gilg, A. W. und Kelly, M.: Rural planning in practice: The case of agricultural dwellings, Prog. Plann., 47, 75-157, https://doi.org/10.1016/S0305-9006(96)00007-4, 1997.

Glass, R. (Hrsg.): London: Aspects of Change, Mac Gibbon and Kee, London, 1964.

Gruber, E., Kobras, M., und Kordel, S.: Lifestyle Migration als Potenzial für ländlich-periphere Gebiete Europas? Die Bewertung wirtschaftlicher und sozialer Auswirkungen aus der Perspektive von Zuwanderern und lokalen Akteuren in Frankreich, Österreich und Spanien, Europa Regional, 24, 16-28, 2017.

Guimond, L. und Simard, M.: Gentrification and neo-rural populations in the Québec countryside: Representations of various actors, J. Rural Stud., 26, 449-464, https://doi.org/10.1016/j.jrurstud.2010.06.002, 2010.

Halfacree, K. H.: Out of place in the Country: travellers and the „rural idyll“, Antipode, 28, 42-72, https://doi.org/10.1111/j.14678330.1996.tb00671.x, 1996.

Hamnett, C.: The Blind Men and the Elephant: The Explanation of Gentrification, Trans. Inst. Br. Geog., 16, 173-189, 1991.

Heeg, S.: Wohnungen als Finanzanlage: Auswirkungen von Responsibilisierung und Finanzialisierung im Bereich des Wohnens, sub \urban. zeitschrift für kritische stadtforschung, 1, 7599, https://doi.org/10.36900/suburban.v1i1.71, 2013.

Heins, S.: Rural living in city and countryside: Demand and supply in the Netherlands, J. Hous. Built Environ., 19, 391-408, https://doi.org/10.1007/s10901-004-3042-4, 2004.

Helbrecht, I.: Urbanität und Ruralität, in: Schlüsselbegriffe der Kultur- und Sozialgeographie, Herausgeber: Lossau, J., Lippuner, R., und Freytag, T., Ulmer, Stuttgart 167-181, 2014.

Heley, J.: The new squirearchy and emergent cultures of the new middle classes in rural areas, J. Rural Stud., 26, 321-331, https://doi.org/10.1016/j.jrurstud.2010.03.002, 2010.

Hines, J. D.: Rural Gentrification as Permanent Tourism: The Creation of the "New" West Archipelago as Postindustrial Cultural Space, Environ. Plan D, 28, 509-525, https://doi.org/10.1068/d3309, 2010a.

Hines, J. D.: In pursuit of experience: The postindustrial gentrification of the rural American West, Ethnography, 11, 285-308, https://doi.org/10.1177/1466138110361846, 2010b.

Holm, A.: Berlin's Gentrification Mainstream, in: The Berlin Reader. A Compendium on Urban Change and Activism, Herausgeber: Bernt, M., Grell, B., und Holm, A., transcript, Bielefeld, 171-187, 2013.

Holm, A.: Gentrification, in: Handbuch Kritische Stadtgeographie, 3. korrigierte und erweiterte Auflage, Herausgeber: Belina, B., Naumann, M., und Strüver, A., Westfälisches Dampfboot, Münster, 152-157, 2018.

Holm, A. und Schulz, G.: GentriMap: A Model for Measuring Gentrification and Displacement, in: Gentrification and Resistance, Herausgeber: Helbrecht, I., Springer VS, Wiesbaden, 251-277, 2018.

Job, H. und Mayer, M. (Hrsg.): Tourismus und Regionalentwicklung in Bayern, Akademie für Raumforschung und Landesplanung, Hannover, 2013.

Kallert, A. and Dudek, S.: ,Aktivieren statt Alimentieren“: Austerität als Paradigma ländlicher Entwicklung am Beispiel Bayern, in: Kritische Geographien ländlicher Entwicklung. Globale Transformationen und lokale Herausforderungen, Herausgeber: Mieß- ner, M. und Naumann, M., Westfälisches Dampfboot, Münster, 177-191, 2019.

Kordel, S. und Pohle, P.: International Lifestyle Migration in the Andes of Ecuador: How Migrants from the USA Perform Privilege, Import Rurality and Evaluate Their Impact on Local Community, Sociol. Rural., 58, 126-146, https://doi.org/10.1111/soru.12133, 2018.

Kröcher, U.: Der New Regionalism: Aufstieg und Fall(stricke) einer regionalwissenschaftlichen Alternative zur Neoklassik, in: Kritische Regionalwissenschaft. Gesellschaft, Politik, Raum Theorien und Konzepte im Überblick, Herausgeber: Krumbein, W., Frieling, H.-D.v., Kröcher, U., und Sträter, D., Westfälisches Dampfboot, Münster, 191-211, 2008.

Krüger, R.: Peripherie 2005: Tourismus und ländlicher Raum, in: Material zur Angewandten Geographie. Band 24, Herausgeber: Moll, P., 55-64, Deutscher Verband für Angewandte Geographie, Bonn, 1995.

Leebrick, R. A.: Rural Gentrification and Growing Regional Tourism: New Development in South Central Appalachia, in: States and Citizens: Accommodation, Facilitation and Resistance to Globalization, Herausgeber: Shefner, J., Emerald, Bingley, 215-234, https://doi.org/10.1108/S0278-120420150000034009, 2015.

Lees, L., Slater, T., und Wyly, E. K.: Gentrification, Routledge, New York, 2008.

Lembke, J.: „Das Dorf muss gentrifiziert werden“, in: Frankfurter Allgemeine Zeitung vom 11. August 2019.

Lier, B.: Eine Studie über die Relevanz ländlicher Gentrification in Deutschland - oder die Suche nach der Nadel im Heuhaufen, Diplomarbeit, TU Dresden, Institut für Geographie, 2012.

Little, J.: Rural-Gentrification and the Influence of Local-level Planning, in: Rural Planning. Policy into Action?, Herausgeber: Cloke, P., 185-199, Harper and Row, London, 1987.

Lopez-Morales, E.: A rural gentrification theory debate for the Global South?, Dialogues Hum. Geogr., 8, 47-50, https://doi.org/10.1177/2043820617752005, 2018.

Mamonova, N. und Sutherland, A.-L.: Rural gentrification in Russia: Renegotiating identity, alternative food production and social tensions in the countryside, J. Rural Stud., 42, 154-165, https://doi.org/10.1016/j.jrurstud.2015.10.008, 2015.

Marcuse, P.: Gentrification, Abandonment, and Displacement: Connections, Causes, and Policy Responses in New York City, U. J. Urb. \& Contemp. L., 28, 195-240, 1985.

Marx, K.: Das Kapital. Kritik der politischen Ökonomie, Band I: Der Produktionsprozess des Kapitals, Dietz, Berlin, 1962[1867].

Maschke, L., Mießner, M., und Naumann, M.: Kritische Landforschung. Konzeptionelle Zugänge, empirische Problemlagen und politische Perspektiven, transcript, Bielefeld, 2020.

Mießner, M.: Staat - Raum - Ordnung. Zur raumordnungspolitischen Regulation regionaler Disparitäten, Westfälisches Dampfboot, Münster, 2017.

Miessner, M.: Studentification in Germany: How Investors Generate Profits from Student Tenants and the Impacts on Urban Segregation, Eu. Urban Reg. Stud., 28, 133-154, https://doi.org/10.1177/0969776420934850, 2021.

Mießner, M.: Angewandte Kritische Geographie am Beispiel der Wohnungsmarktforschung in Göttingen, Standort, 44, 239-247, https://doi.org/10.1007/s00548-020-00665-8, 2020. 
Nelson, L. und Nelson, P. B.: The global rural: Gentrification and linked migration in the rural USA, Prog. Hum. Geog., 35, 441459, https://doi.org/10.1177/0309132510380487, 2011.

Newby, H.: The Deferential Worker: A Study of Farm Workers in East Anglia, Penguin education, Harmondsworth, Middlesex, 1979.

Parsons, D.: Rural Gentrification: The Influence of Rural Settlement Planning Policies, University of Sussex, Brighton, University of Sussex research papers in geography, 3, 1980.

Phillips, M.: Rural gentrification and the processes of class colonisation, J. Rural Stud., 9, 123-140, https://doi.org/10.1016/07430167(93)90026-G, 1993.

Phillips, M.: Other geographies of gentrification, Prog. Hum. Geog., 28, 5-30, https://doi.org/10.1191/0309132504ph458oa, 2004.

Phillips, M.: Gentrification, Rural, in: International Encyclopedia of Human Geography, Elsevier, Amsterdam, 368-375, https://doi.org/10.1016/B978-008044910-4.00899-3, 2009.

Phillips, M.: Counterurbanisation and rural gentrification: an exploration of the terms, Popul. Space Place, 16, 539-558, https://doi.org/10.1002/psp.570, 2010.

Phillips, M.: Baroque rurality in an English village, J. Rural Stud., 33, 56-70, https://doi.org/10.1016/j.jrurstud.2013.10.008, 2014.

Phillips, M., Page, S., Saratsi, E., Tansey, K., und Moore, K.: Diversity, scale and green landscapes in the gentrification process: Traversing ecological and social science perspectives, Appl. Geogr., 28, 54-76, https://doi.org/10.1016/j.apgeog.2007.07.003, 2008.

Phillips, M. und Smith, D.: Comparative approaches to gentrification: Lessons from the rural, Dialogues Hum. Geog., 8, 3-25, https://doi.org/10.1177/2043820617752009, 2018.

Phillips, M., Smith, D., Brooking, H., und Duer, M.: Re-placing displacement in gentrification studies: Temporality and multidimensionality in rural gentrification displacement, Geoforum, 118, 66-82, https://doi.org/10.1016/j.geoforum.2020.12.003, 2021.

Pichler, H.: Die Renaissance des Wohnstandortes Dorf. Zum Phänomen der Gentrification im ländlichen Raum am Beispiel der burgenländischen Gemeinde Breitenbrunn, geographie heute, 19, 20-24, 1998.

Porter, M.: The Rent Gap at the Metropolitan Scale: New York City's Land-Value Valleys, 1990-2006, Urban Geogr., 31, 385405, https://doi.org/10.2747/0272-3638.31.3.385, 2010.

Rat der Immobilienweisen: Frühjahrsgutachten Immobilienwirtschaft 2020, Zentraler Immobilienausschuss e.V., Berlin, 2020.

Reichert-Schick, A.: Rural gentrification an der Obermosel? Eine Fallstudie zu internationaler Wohnmigration und den Herausforderungen für die Dorfentwicklung, Europa Regional, 24, 77-94, 2017.

Richard, F., Dellier, J., und Tommasi, G.: Migration environment and rural gentrification in the Limousin mountains, Journal of Alpine Research, 102, 1-15, https://doi.org/10.4000/rga.2561, 2014.

Robinson, G. M.: Conflict and Change in the Countryside. Rural Society, Economy and Planning in the Developed World, Wiley, West Sussex, 1996.

Robinson, J. und Roy, A.: Debate on Global Urbanisms and the Nature of Urban Theory, Int. J. Urban Regional, 40, 181-186, https://doi.org/10.1111/1468-2427.12272, 2016.

Rohr, G. v. (Hrsg.): Nachhaltiger Tourismus an der Nord- und Ostsee. Steuerungsnotwendigkeiten und -möglichkeiten der Landes- und Regionalplanung, Akademie für Raumordnung und Landesplanung, Hannover, 2008.

Rose, D.: Rethinking Gentrification: Beyond the Uneven Development of Marxist Urban Theory, Environ. Plan. D, 2, 47-74, https://doi.org/10.1068/d020047, 1984.

Schipper, S.: Global-City-Formierung, Gentrifizierung und Grundrentenbildung in Frankfurt am Main, Z. Wirtsch., 57, 185-200, https://doi.org/10.1515/zfw.2013.0014, 2013.

Schmidt, W.: Luxus Landleben. Neue Ländlichkeit am Beispiel Mecklenburgs, Mecklenburger AnStiftung, Wismar, 2017.

Schmied, D.: What Price Piece and Quiet? Rural Gentrification and Affordable Housing. The Example of Cotswold District, South West England, Naturwissenschaftliche Gesellschaft Bayreuth, Bayreuth, 2002.

Schönig, B.: Unter dem Radar. Wohnungsfragen abseits der Wachstumsräume in Thüringer Klein- und Mittelstädten, in: Wohnungsfragen ohne Ende?! Ressourcen für eine soziale Wohnraumversorgung, Herausgeber: Schönig, B. und Vollmer, L., transcript, Bielefeld, 207-229, https://doi.org/10.14361/9783839445082-013, 2020.

Scott, M., Smith, D., Shucksmith, M., Gallent, N., Halfacree, K., Kilpatrick, S., Johns, S., Vitartas, P., Homisan, M., und Cherrett, T.: Interface. Exclusive Countrysides? Rural Gentrification, Consumer Preferences and Planning Theory \& Practice, 12, 593 635, https://doi.org/10.1080/14649357.2011.626304, 2011.

Shucksmith, M.: No Homes for Locals?, University of Michigan, Gower, 1981.

Shucksmith, M.: Exclusive Rurality: Planners as Agents of Gentrification, Planning Theory \& Practice, 12, 605-611, 2011.

Simmank, M.: Wohnen auf dem Land: Infrastrukturen für "gleichwertige Lebensverhältnisse“, in: Wohnungsfragen ohne Ende?! Ressourcen für eine soziale Wohnraumversorgung, Herausgeber: Schönig, B. und Vollmer, L., transcript, Bielefeld, 193-205, https://doi.org/10.14361/9783839445082-012, 2020.

Slater, T.: The Eviction of Critical Perspectives from Gentrification Research, Int. J. Urban Regional, 30, 737-757, https://doi.org/10.1111/j.1468-2427.2006.00689.x, 2006.

Smith, D. P.: The 'Buoyancy' of 'Other' Geographies of Gentrification: Going 'Back-to-the Water' and the Commodification of Marginality, Tijdschr. Econ. Soc. Ge., 98, 53-67, https://doi.org/10.1111/j.1467-9663.2007.00376.x, 2007.

Smith, D. P.: What is Rural Gentrification?: Exclusionary Migration, Population Change, and Revalorised Housing Markets, Planning Theory \& Practice, 12, 596-605, https://doi.org/10.1080/14649357.2011.626304, 2011.

Smith, D. P. und Higley, R.: Circuits of education, rural gentrification, and family migration from the global city, J. Rural Stud., 28, 49-55, https://doi.org/10.1016/j.jrurstud.2011.08.001, 2012.

Smith, D. P. und Holt, L.: 'Lesbian migrants in the gentrified valley' and 'other' geographies of rural gentrification, J. Rural Stud., 21, 313-322, https://doi.org/10.1016/j.jrurstud.2005.04.002, 2005.

Smith, D. P. und Phillips, D. A.: Socio-cultural representations of greentrified Pennine rurality, J. Rural Stud., 17, 457-469, https://doi.org/10.1016/S0743-0167(01)00014-6, 2001.

Smith, N.: Für eine Theorie der Gentrifizierung, sub $\backslash$ urban. zeitschrift für kritische stadtforschung, 7, 65-86, https://doi.org/10.36900/suburban.v7i3.546, 2019 [1979].

Smith, N.: The New Urban Frontier: Gentrification and the Revanchist City, Routledge, London, New York, 1996. 
Smith, N.: New Globalism, New Urbanism: Gentrification as Global Urban Strategy, Antipode, 34, 427-450, https://doi.org/10.1111/1467-8330.00249, 2002.

Solana-Solana, M.: Rural gentrification in Catalonia, Spain: A case study of migration, social change and conflicts in the Empordanet area, Geoforum, 41, 508-517, https://doi.org/10.1016/j.geoforum.2010.01.005, 2010.

Spencer, D.: Counterurbanisation and rural depopulation revisited: Landowners, planners and the rural development process, J. Rural Stud., 13, 75-92, https://doi.org/10.1016/S07430167(96)00059-9, 1997.

Steel, G., van Noorloos, F., und Klaufus, C.: The urban land debate in the global South: new avenues for research, Geoforum, 83, 133-141, https://doi.org/10.1016/j.geoforum.2017.03.006, 2017.

Stockdale, A.: The diverse geographies of rural gentrification in Scotland, J. Rural Stud., 26, 31-40, https://doi.org/10.1016/j.jrurstud.2009.04.001, 2010.

Stockdale, A.: Unravelling the migration decision-making process: English early retirees moving to rural mid-Wales, J. Rural Stud., 34, 161-171, https://doi.org/10.1016/j.jrurstud.2014.01.010, 2014.
Sutherland, L.-A.: Return of the gentleman farmer?: Conceptualising gentrification in UK agriculture, J. Rural Stud., 28, 568-576, https://doi.org/10.1016/j.jrurstud.2012.05.001, 2012.

Thünen-Institut für Ländliche Räume: Landatlas, verfügbar unter: https://www.landatlas.de/ (letzter Zugriff: 3 Mai 2021), Ausgabe 2020, Braunschweig, 2020.

Vergunst, J.: Changing Environmental Values. Beyond Production and Consumption, in: Routledge International Handbook of Rural Studies, Herausgeber: Shucksmith, M. und Brown, D. L., Routledge, Abingdon, 285-294, 2016.

Visser, G.: Second homes and local development: Issues arising from Cape Town's De Waterkant, GeoJournal, 60, 259-271, https://doi.org/10.1023/B:GEJO.0000034733.80648.88, 2004.

Witzeck, E.: Viel Wald, wenig Kompromisse, in: Frankfurter Allgemeine Zeitung vom 25. Mai 2020.

Wright, E. O.: Class, Crisis and the State, Verso, London, 1993 [1978]. 